\title{
Chondrosarcoma of T2 Vertebrae Treated by Combined Anterior and Posterior Approach: A Case Report
}

\author{
K Norazian, MBBS, LB Saw, MS Ortho, CYW Chan, MS Ortho, R Amin*, MS Surg, MK Kwan, MS Ortho \\ Department of Orthopaedic Surgery, *Department of Surgery, \\ Faculty of Medicine, University of Malaya, Kuala Lumpur, Malaysia
}

\begin{abstract}
Chondrosarcoma of the spine is rare; it presents predominantly in very young males and presentation with neurological deficit is uncommon. Treatment of this type of tumour is mainly through surgery as adjuvant therapy is ineffective. En bloc resection of tumours in the spine are difficult although it remains the recommended treatment for chondrosarcoma. We report here presentation of a female with paresis (Frankel C) whom was diagnosed with a large chondrosarcoma of the T2 vertebra extending to the right upper thoracic cavity. The patient underwent radical excision through an anterior and posterior approach to the spine.
\end{abstract}

Key Words:

Chondrosarcoma, T2 Vertebrae

\section{INTRODUCTION}

Chondrosarcomas, malignant lesions, represent the third most common tumour of the skeleton after osteosarcoma and Ewing's sarcoma ${ }^{1}$. However, chondrosarcoma presenting as a primary tumour of the spine is extremely rare. These tumours can originate from healthy bone or develop from a cartilaginous lesion with sarcomatous changes. The majority of patients with chondrosarcoma are older than 50 years, with peak incidence in the fifth to seventh decade of life.

Chondrosarcomas of the spinal column are most frequently found in the thoracic spine ${ }^{1}$. Herein we present a 33 year-old Malay woman presenting with paraparesis (Frankel C) who was diagnosed with a large chondrosarcoma of the T2 vertebra extending to the right upper thoracic cavity.

\section{CASE REPORT}

A 33-year-old woman referred from an outlying hospital, presented with progressive bilateral lower limb weakness, which resulted in an inability to walk for two weeks prior to admission. There was no bowel and bladder dysfunction. The patient also complained of persistent upper thoracic pain for the last three years, which was treated with nonsteroidal anti-inflammatory drugs and physiotherapy. The pain was nonmechanical, burning in nature and worse at night. She had no other associated constitutional symptoms such as fever, cough, loss of appetite or weight loss and no history of trauma.

Physical examination showed tenderness at the cervicothoracic region. There was no palpable lump, spine deformity or skin discolouration noted. On neurological examination, there was complete loss of motor function of the left lower extremity and only grade 2 motor function of the right lower extremity with hypoaesthesia below the T4 level. Deep tendon reflexes of bilateral lower limbs showed hyperreflexia with bilateral positive Babinski reflexes.

Blood work did not show any abnormality. Inflammatory markers (i.e., C-reactive protein and erythrocyte sedimentation rate) were normal with no increase in total white cell and normal serum bone profiles. All tumour markers were within normal range. Anteroposterior plain radiograph of the thoracic spine showed an osteolytic lesion and destruction of both T2 vertebra pedicles with mild collapse of the vertebral body and a large homogenous mass in the upper region of the right lung. No obvious calcification was seen on the plain radiograph. Magnetic resonance imaging (MRI) showed a well-demarcated tumour mass measuring $7.5 \times 9.0 \times 8.5 \mathrm{~cm}$ in the upper region of the right lung originating from the T2 vertebral body (Figure 1). The tumour compressed and displaced the trachea to the left, damaging the T2 vertebral body and invading the spinal canal causing spinal cord compression. On T1-weighted images, the tumour showed areas of low homo-intensity signal and heterogeneous low and high intensities, and mineralized as well as non-mineralized parts on T2-weighted images.

A CT-guided needle biopsy was performed; resulting histopathological examination revealed an aggressive tumour with calcification and presence of marked myxoid degeneration suggestive of well differentiated grade II chondrosarcoma. The patient was diagnosed with spinal cord compression with Frankel $\mathrm{C}$ neurology due to high grade 

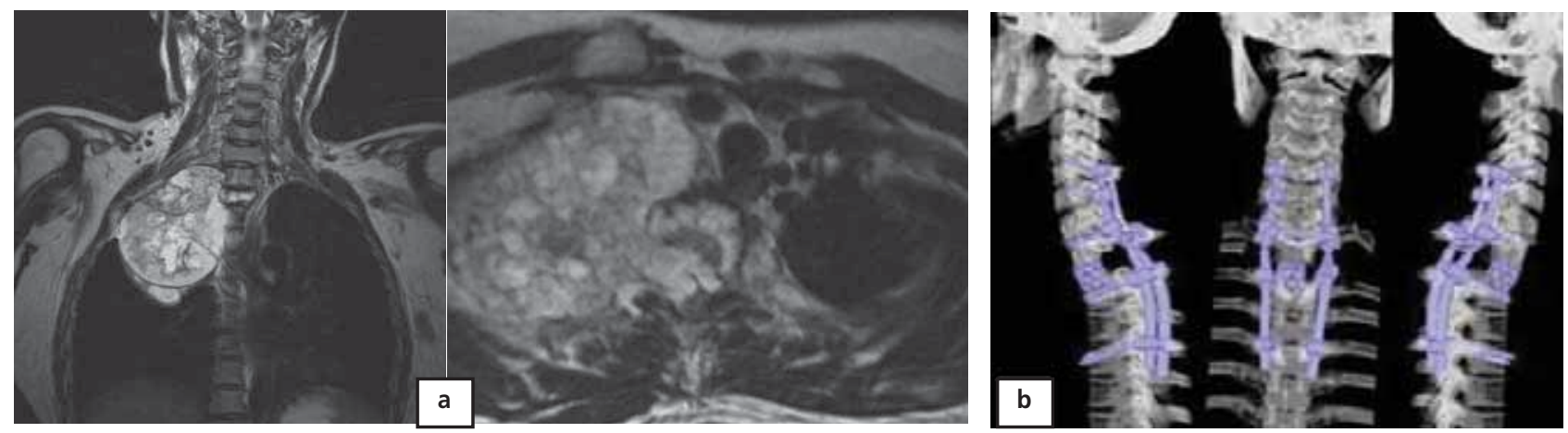

Fig. 1: (A) T2-weighted MR image (coronal view) showed a high signal heterogenous mass at the upper zone of the right lung originating from the $\mathrm{T} 2$ vertebral body. The heterogenous tumor also invaded the spinal cord circumferentially causing narrowing of the canal and compressed the spinal cord. (B) Post operative CT scan to evaluate resection margin and reconstruction of the spinal column.

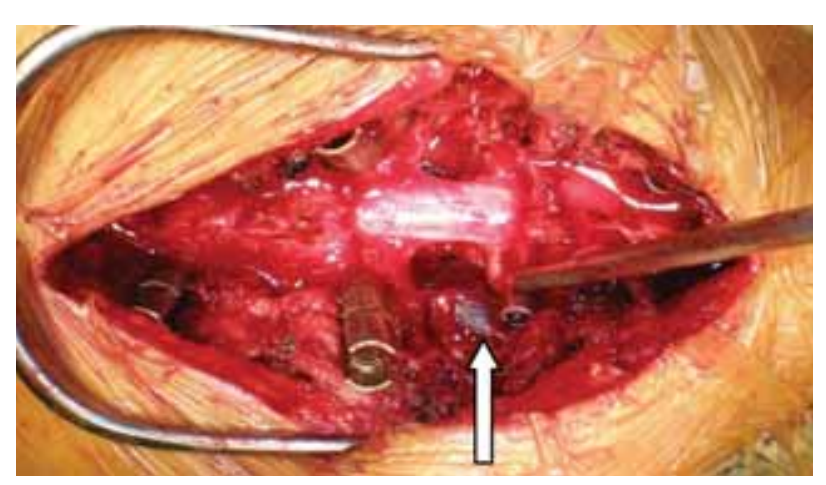

Fig. 2: Exposed dura after decompression and vertebrectomy of T2. Arrow pointing to a vicryl tape to mark the right T1 nerve root and the anterior part of the titanium cage.

extracompartmental (Enneking Stage IIB) T2 chondrosarcoma with massive extension to the right upper thoracic cavity.

A two staged (posterior approach procedure followed by an anterior approach procedure) surgery was planned for the patient. A cardiothoracic surgeon was consulted regarding the large intrathoracic tumour mass in the upper region of the right lung. The first stage was performed via a posterior approach; lateral mass screws were inserted in the C5 and C6 vertebrae and pedicle screw stabilization was performed on the T1, T3 and T5 vertebrae. Direct decompression and vertebrectomy of $\mathrm{T} 2$ was carried out utilizing a costotransversectomy approach. The left $\mathrm{T} 1$ and $\mathrm{T} 2$ nerve root was clearly visualized and protected; then the intervertebral discs of $\mathrm{T} 1 / 2$ and $\mathrm{T} 2 / 3$ were completely removed and anterior column reconstruction was performed using a titanium cage filled with bone grafts placed between the T1 and T3 vertebral bodies. Before wound closure, a Vicryl tape was inserted anterior to the titanium cage and right $\mathrm{T} 1$ nerve root as markers for anterior clearance for the second stage of surgery (Figure 2).
The second surgery was performed one week after the first procedure. Wide resection of the tumour, including part of the upper lobe of the right lung, was performed by the cardiothoracic team. Bone resection was carried out to the level of the Vicryl tape placed during the first procedure to indicate total clearance of the tumour.

The first procedure took 5 hours and incurred blood loss of 2.5 litres, while the second procedure took four hours with total blood of loss two litres. Postoperatively, the patient neurology improved from Frankel C to Frankel D (motor power of grade 4), which enabled ambulation with a walking frame after two weeks. A postoperative CT scan showed complete resection of the tumour with proper placement of the cage and stable posterior and anterior reconstruction (Figure 1b). The patient developed right-sided Horner syndrome from the second procedure caused by traction injury on the truncus sympathicus; partial recovery was seen during follow-up at 3 months. At the 6 months follow-up, neurology status was further improved, as the patient was able to ambulate with a walking stick; she is still being seen regularly for ongoing regular follow-up.

\section{DISCUSSION}

Chondrosarcoma constitutes a family of malignant tumours in which the cells tend to differentiate into cartilage. The prevalence of chondrosarcoma in the spine as a whole is reported to range from $4 \%$ to $10 \%$ of all chondrosarcoma cases $^{1}$, and only a few cases are reported in the literature ${ }^{2}$. The thoracic spine is the most common location for spinal chondrosarcoma, and it occurs predominantly in males ${ }^{3,4}$. Radiologically, chondrosarcomas are typically seen as a large area of bone destruction and a soft tissue mass having flocculent calcifications within, but this was not obvious in the radiographs of our patient. In some cases, there is no soft tissue mass, the vertebral lesion may be primarily lytic with sclerotic margins and no mottled calcification ${ }^{4}$. 
The clinical course of chondrosarcoma originating in the spine can be prolonged because the tumour grows slowly; this was true in the present case as the patient had upper thoracic pain for the past three years. Frequently, the posterior arch is involved causing a palpable mass; neurological weakness at first presentation is relatively uncommon as it rarely grows inside the spinal canal ${ }^{4}$. However, this patient presented with paraparesis due to spinal cord compression from chondrosarcoma of the T2 vertebrae extending to the spinal canal and thoracic cavity.

Treatment with wide excision for chondrosarcoma in the limbs is clearly established and commonly accepted. Prognosis is dependent upon the histological grade of malignancy and on the feasibility of performing en bloc excision with appropriate oncologic margins. On the other hand, surgical treatment in spinal chondrosarcoma is particularly difficult, because of the difficulties in executing en bloc excision in the spinal column. This patient demonstrated a well differentiated grade II chondrosarcoma with good prognosis, as good oncologic surgical clearance was achievable. However, due to difficulties associated with en bloc surgery in the spine, lesions in the vertebral column can have a poor prognosis independent of the histological grade. In this case, spinal cord decompression and intralesional removal of the tumour including margins free from the tumour were performed.

As expected, local recurrence is higher in patients who undergo piecemeal excision. York et al. evaluated 21 patients with spinal chondrosarcoma over a period of 43 years, and reported a longer disease-free interval in patients who underwent total resection compared to those who underwent subtotal resection ${ }^{2}$. The recurrence rate for piecemeal excision was $64 \%$ and the median disease free interval was 16 months without adjuvant therapy ${ }^{2}$. Shives et al. studied 20 patients with chondrosarcoma of the spine and found $100 \%$ recurrence in patients submitting to tumour debulking only, $50 \%$ recurrence in six patients who underwent tumour excision but with inappropriate surgical margins and no recurrences in two patients who had radical resection ${ }^{3}$. Tumour contamination of specimen margins or spreading of tumour myxoid content has been shown to worsen the prognosis of the surgery ${ }^{4}$. In the present case, the tumour occupied the whole right upper lung cavity; thus, it was too large to remove through anterior or/and posterior en bloc resection surgery alone. We therefore undertook a two staged posterior and then anterior intralesional piecemeal surgery with appropriate oncologic margin of resection. In this case, the key step to achieving a good oncologic margin after the posterior vertebrectomy was proper tagging of the posterior excision margin with the use of Vicryl tape, so that the margin of the posterior excision could be visualized during the anterior resection to ensure adequate oncologic clearance of the tumour despite use of the intralesional procedure due to the size of the tumour mass.

The use of postoperative radiotherapy in the treatment of chondrosarcoma is controversial. Patients submitting to radiotherapy do not demonstrate a significant increase in survival in relation to those treated by surgery alone ${ }^{2}$. There are limited studies in the literature that demonstrate efficacy of radiotherapy or chemotherapy in spinal chondrosarcoma. Kim et al. reported the results of intralesional excision and then high energy irradiation as an adjuvant therapy to prevent local recurrences in skeletal chondrosarcoma ${ }^{5}$. In three of the five reported cases, long-term local control was obtained, although two patients (with lumbar and sacral chondrosarcoma) died of local recurrence 3 years after radiotherapy. Therefore, total resection of the tumour provides the best chance for prolonging the disease-free interval in patients with chondrosarcoma.

\section{CONCLUSION}

Surgery remains an effective treatment for localized chondrosarcoma of the spine. En bloc resection of the vertebral body (vertebrectomy) may be curative when it is possible to remove the whole tumour along with a continuous, even and thin shell of normal tissue.

\section{ACKNOWLEDGEMENT}

I would like to take this opportunity to express my appreciation Hew Yook Ping and Shirley Wong Kueng Chii for their invaluable help in data collection and manuscript preparation. 
Treatment of Chondrosarcoma of the T2 Vertebra Using a Combined Anterior and Posterior Approach

\section{REFERENCES}

1. Campanacci P. Bone and Soft Tissues Tumors. New York: New York and Bologna: Springer-Verlag; 1990: 414-5.

2. York JE, Berk RH, Fuller GN, Rao JS, Abi-Said D, Wildrick DM et al. Chondrosarcoma of the spine: 1954 to 1997. J Neurosurg 1999; 90: 73-8.

3. Shives TC, McLeod RA, Unni KK, Schray MF. Chondrosarcoma of the spine. J Bone Joint Surg Am 1989; 71(8): $1158-65$.

4. Boriani S, De lure F, Bandiera S, Campanaci L, Biagini R, Di Fiore M, et al. Chondrosarcoma of the mobile spine. Report on 22 cases. Spine 2000; 25(7): 804-12.

5. Kim RJ, Salter MM, Brascho DJ. High-energy irradiation in the management of chondrosarcoma. South Med J 1983; 76: 729-31. 